\title{
The blue host galaxy of the red GRB 000418
}

J. Gorosabel ${ }^{1,2,3}$, S. Klose ${ }^{4}$, L. Christensen ${ }^{5}$, J. P. U. Fynbo ${ }^{6,7}$, J. Hjorth ${ }^{7}$, J. Greiner ${ }^{8}$, N. Tanvir ${ }^{9}$, B. L. Jensen ${ }^{7}$, H. Pedersen ${ }^{7}$, S. T. Holland ${ }^{10}$, N. Lund ${ }^{2}$, A. O. Jaunsen ${ }^{11}$, J. M. Castro Cerón ${ }^{12}$, A. J. Castro-Tirado ${ }^{1}$, A. Fruchter ${ }^{3}$, E. Pian ${ }^{13}$, P. M. Vreeswijk ${ }^{11}$, I. Burud ${ }^{3}$, F. Frontera ${ }^{14,15}$, L. Kaper ${ }^{16}$, C. Kouveliotou ${ }^{17}$, N. Masetti ${ }^{15}$, E. Palazzi ${ }^{15}$, J. Rhoads ${ }^{3}$, E. Rol ${ }^{16}$, I. Salamanca ${ }^{16}$, R. A. M. J. Wijers ${ }^{16}$, and E. van den Heuvel ${ }^{16}$

${ }^{1}$ Instituto de Astrofísica de Andalucía (IAA-CSIC), PO Box 03004, 18080 Granada, Spain; e-mail: jgu@iaa.es, ajct@iaa.es

2 Danish Space Research Institute, Juliane Maries Vej 30, 2100 Copenhagen Ø, Denmark e-mail: jgu@dsri.dk, nl@dsri.dk

3 Space Telescope Science Institute, 3700 San Martin Drive, Baltimore, MD 21218, USA e-mail: gorosabel@stsci.edu, fruchter@stsci.edu, burud@stsci.edu, rhoads@stsci.edu

4 Thüringer Landessternwarte Tautenburg, 07778 Tautenburg, Germany; e-mail: klose@tls-tautenburg.de

5 Astrophysikalisches Institut, 14482 Potsdam, Germany; e-mail: 1christensen@aip.de

${ }^{6}$ Department of Physics and Astronomy, University of Aarhus, Ny Munkegade, 8000 Århus C, Denmark e-mail: jfynbo@phys.au.dk

7 Astronomical Observatory, University of Copenhagen, Juliane Maries Vej 30, 2100 Copenhagen Ø, Denmark e-mail: jens@astro.ku.dk, brian_j@astro.ku.dk, holger@astro.ku.dk

8 Max-Planck-Institut für extraterrestrische Physik, 85741 Garching, Germany; e-mail: jcg@mpe.mpg.de

9 Department of Physical Sciences, University of Hertfordshire, College Lane, Hatfield, Herts AL10 9AB, UK e-mail: nrt@star.herts.ac.uk

10 Department of Physics, University of Notre Dame, Notre Dame, IN 46556-5670, USA; e-mail: sholland@nd. edu

11 European Southern Observatory, Casilla 19001, Santiago 19, Chile; e-mail: ajaunsen@eso.org, pvreeswi@eso.org

12 Real Instituto y Observatorio de la Armada, Sección de Astronomía, 11.110 San Fernando-Naval, Cádiz, Spain e-mail: josemari@alumni.nd.edu

13 Osservatorio Astronomico di Trieste, via G.B. Tiepolo 11, 34131, Trieste, Italy; e-mail: pian@tesre.bo.cnr.it

14 Dipartimento di Fisica, Università di Ferrara, via Paradiso 12, 44100 Ferrara, Italy; e-mail: frontera@fe.infn.it

15 Istituto Tecnologie e Studio Radiazioni Extraterrestri, CNR, via Gobetti 101, 40129 Bologna, Italy e-mail: filippo@bo.iasf.cnr.it, masetti@bo.iasf.cnr.it, eliana@bo.iasf.cnr.it

16 University of Amsterdam, Kruislaan 403, 1098 SJ Amsterdam, The Netherlands e-mail: lexk@science.uva.nl, evert@science.uva.nl,isabel@science.uva.nl, rwijers@science.uva.nl, edvdh@science.uva.nl

17 NASA MSFC, SD-50, Huntsville, AL 35812, USA; e-mail: kouveliotou@eagles.msfc.nasa.gov

Received 30 April 2003 / Accepted 7 July 2003

\begin{abstract}
We report on multi-band $\left(U B V R I Z J_{\mathrm{s}} K_{\mathrm{s}}\right)$ observations of the host galaxy of the April 18, 2000 gamma-ray burst. The Spectral Energy Distribution (SED) is analysed by fitting empirical and synthetic spectral templates. We find that: (i) the best SED fit is obtained with a starburst template, (ii) the photometric redshift is consistent with the spectroscopic redshift, (iii) the colours of the host are inconsistent with an old stellar population, and (iv) the global extinction is constrained to be in the range $A_{V}=0.12-0.61 \mathrm{mag}$. The derived global extinction agrees with the one reported for the afterglow $\left(A_{V}=0.4-0.9 \mathrm{mag}\right)$, suggesting a homogeneous distribution of the interstellar medium (ISM) in the host galaxy. These findings are supplemented by morphological information from Hubble Space Telescope (HST) imaging: the surface brightness profile is smooth, symmetric and compact with no underlying structures (like dust lanes, spiral arms or disks). A natural scenario which accounts of all the above results is a nuclear starburst that harbours a young population of stars from which the GRB originated.
\end{abstract}

Key words. gamma rays: bursts - galaxies: fundamental parameters - techniques: photometric

Send offprint requests to: J. Gorosabel, e-mail: jgu@iaa.es

^ Based on observations made with the Nordic Optical Telescope, operated on the island of La Palma jointly by Denmark, Finland, Norway, and Sweden. Based on observations collected at the European Southern Observatory, in La Silla and Paranal (Chile), ESO
Large Programmes 165.H-0464(I) and 265.D-5742(C), granted to the GRACE Team. Based on public data collected under ESO programme 67.B-0611(A), and retrieved from ESO data archive. Based on HST data collected under programme 8189. 


\section{Introduction}

Since $1997 \sim 40$ gamma-ray burst (GRB) optical afterglows (OAs) have been discovered (see the GRB compilation of $\mathrm{J}$. Greiner ${ }^{1}$ ). GRBs generally occur in subluminous host galaxies with redshifts ranging from $z=0.1685$ (GRB 030329; Hjorth et al. 2003) to $z=4.50$ (GRB 000131; Andersen et al. 2000). Most of the GRB hosts are subluminous and have bluer optical/near-IR colours than the local galaxies or the typical galaxies detected by the Infrared Space Observatory (ISO) and the Submillimeter Common-User Bolometer Array (SCUBA) (e.g. Le Floc'h et al. 2003).

The afterglow of GRB 000418 was discovered in the nearinfrared (NIR) 2.5 days after the gamma-ray event (Klose et al. 2000a). The optical counterpart was rather faint $(R=21.63$, 2.48 days after the GRB) in comparison to other afterglows (see Fig. 3 of Gorosabel et al. 2002a for comparison purposes). The $R$-band light curve decayed as $t^{-1.22}$ typical of OAs (Klose et al. 2000b), flattening off at a level of $R \sim 24$ due to the underlying host galaxy (Bloom et al. 2000). The afterglow is one of the reddest $(R-K=4)$ detected to date (see Fig. 2 of Gorosabel et al. 2002b). Klose et al. (2000b) suggested that the red colour is caused by reddening due to dust extinction in the host galaxy and they estimated an extinction of $A_{V} \sim 0.9$ mag. Berger et al. (2001) found $A_{V} \sim 0.4 \mathrm{mag}$ for the OA.

HST/STIS observations performed on 4.17 UT June 2000 (46.76 days after the GRB) revealed that the OA occurred in a very compact host galaxy with a half-light radius of $\sim 0$ '. $^{\prime} 13$ (Metzger et al. 2000) corresponding to about $1 \mathrm{kpc}$. The redshift of the host galaxy was determined to be $z=1.118$ (Bloom et al. 2002, 2003). A preliminary $B V R I$-band SED fitting analysis showed that the host galaxy SED can be fitted with starburst galaxy templates, but not with an evolved stellar population (Gorosabel et al. 2001). This result has been recently supported by Bloom et al. (2003) who based on the optical emission lines suggest that the host is a starburst galaxy, rather than a LINER or a Seyfert 2 galaxy. Bloom et al. (2003) estimate an unobscured star formation rate (SFR) of $55 M_{\odot} \mathrm{yr}^{-1}$ based on the [O II] emission line diagnostic method (Kennicutt 1992).

Berger et al. (2003) and Barnard et al. (2003) have recently reported tentative detections of several GRB host galaxies in the sub-millimeter and radio ranges, inferring SFRs of hundreds of Solar masses per year. The most significant detection was from the host galaxy of GRB 000418 for which the sub$\mathrm{mm}$ detection is significant at the $3.6 \sigma$ level and the radio emission from the host again at more than $3 \sigma$. Berger et al. (2003) also detected another faint radio source only 1.4 arcsec from the GRB 000418 host. This source is not seen in the optical down to $R>27$. Assuming that the sub-mm and radio emission is due to dust heated by star formation Berger et al. (2003) derived SFRs of $690 \pm 195$ and $330 \pm 75 M_{\odot} \mathrm{yr}^{-1}$ respectively, i.e. much higher than the SFR derived from the optical emission lines by Bloom et al. (2003).

In this paper we present a comprehensive multicolour study of the GRB 000418 host galaxy, similar to those performed for the GRB 000210 (Gorosabel et al. 2003) and GRB 990712

\footnotetext{
${ }^{1}$ http://www.mpe.mpg.de/ jcg/grbgen.html
}

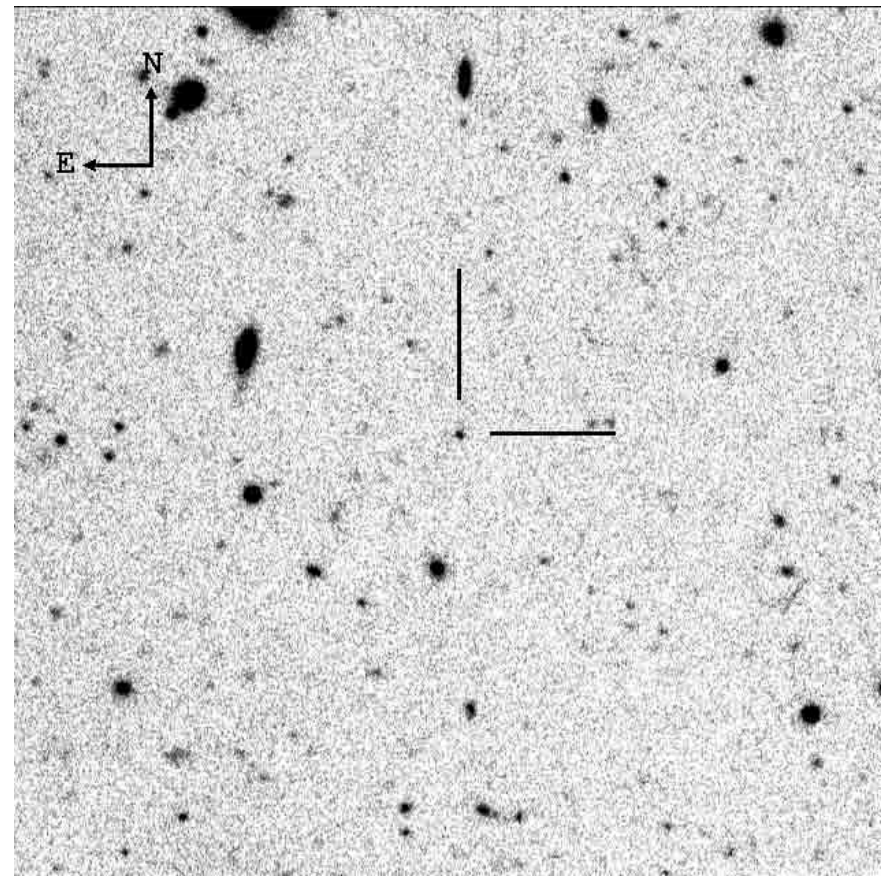

Fig. 1. The image shows the coadded $V$-band image taken with the 2.5 NOT at 31.067-31.135 March 2001 UT. The FOV covered by the image corresponds to $2^{\prime} .0 \times 2$ '. 0 . North is to the top and East to the left.

(Christensen et al. 2003) host galaxies. The aim of the analysis is to determine the properties of the stellar populations dominating the optical/near-IR light from the host galaxy and the amount of extinction due to dust in the interstellar medium (ISM) of the host. Other multi-colour host galaxies studies to date (Sokolov et al. 2001; Chary et al. 2002; Gorosabel et al. 2001) have been limited to a smaller number of bands.

Throughout, the assumed cosmology will be $\Omega_{\Lambda}=0.7$, $\Omega_{M}=0.3$ and $H_{0}=65 \mathrm{~km} \mathrm{~s}^{-1} \mathrm{Mpc}^{-1}$. At the spectroscopic redshift of the host galaxy $(z=1.118)$, the look back time is $8.78 \mathrm{Gyr}(\approx 60 \%$ of the present age $)$ and the luminosity distance is $8.17 \mathrm{Gpc}$. The physical transverse size of one arcsec at $z=1.118$ corresponds to $8.83 \mathrm{kpc}$.

\section{Observations and photometry}

We have used ground-based and space optical/NIR resources in order to sample the host galaxy SED in as many filters as possible over a wide spectral range (see Table 1). The observations were performed 299-676 days after the gamma-ray event. At these times the contribution of the afterglow is negligible and does not have any significant impact on the derived host galaxy SED.

\subsection{Ground-based optical and near-infrared observations}

Given the compactness of the GRB 000418 host galaxy, its ground-based NIR and optical profiles are consistent with that of field stars. This fact has been checked by comparing the growth curve (from 0.5 to 4 times their full width half maximum; FWHM hereafter) of the host and the secondary 
Table 1. Chronologically ordered optical and NIR observations carried out for the GRB 000418 host galaxy.

\begin{tabular}{lcccr}
\hline $\begin{array}{l}\text { Telescope } \\
(+ \text { Instrument) }\end{array}$ & Filter & Date UT & $\begin{array}{c}T_{\text {exp }} \\
(\mathrm{s})\end{array}$ & Seeing \\
\hline HST (+STIS) & 50 CCD & $11.638-11.729 / 02 / 01$ & $8 \times 640$ & $\#$ \\
2.5 NOT (+ALFOSC) & $I$ & $30.909-31.043 / 03 / 01$ & $19 \times 300$ & 0.85 \\
2.5 NOT (+ALFOSC) & $V$ & $31.067-31.135 / 03 / 01$ & $6 \times 900$ & 0.95 \\
2.5 NOT (+ALFOSC) & $R$ & $31.921 / 03-1.063 / 04 / 01$ & $18 \times 600$ & $1.10^{\dagger}$ \\
2.5 NOT (+ALFOSC) & $B$ & $1.064-1.189 / 04 / 01$ & $8 \times 1200$ & 1.15 \\
2.5 NOT (+ALFOSC) & $Z$ & $28.901-28.973 / 05 / 01$ & $15 \times 300$ & $0.85^{\star}$ \\
2.5 NOT (+ALFOSC) & $Z$ & $29.899-29.918 / 05 / 01$ & $4 \times 300$ & $1.20^{\star}$ \\
2.5 NOT (+ALFOSC) & $Z$ & $30.897-30.963 / 05 / 01$ & $14 \times 300$ & $1.10^{\star}$ \\
8.2 VLT (+ISAAC) & $K_{\mathrm{s}}$ & $15.000-15.054 / 06 / 01$ & $30 \times 120$ & 1.20 \\
8.2 VLT (+ISAAC) & $J_{\mathrm{s}}$ & $4.366-4.391 / 02 / 02$ & $15 \times 120$ & 0.90 \\
3.6 ESO (+EFOSC2) & $U$ & $23.310-23.357 / 02 / 02$ & $6 \times 600$ & 1.30 \\
\hline \# Not applicable. & & & & \\
$\dagger$ \\
Through cirrus.
\end{tabular}

Table 2. Magnitudes of the host in the STIS $C L$ and the ground-based $U B V R I Z J_{\mathrm{s}} K_{\mathrm{s}}$ bands. Several characteristics of the filters are displayed: filter name (1), effective wavelength (2) and bandpass width (3). The fourth column shows the measured magnitudes (in the Vega system and corrected from Galactic reddening, considering $E(B-V)=0.033$ given by Schlegel et al. 1998). To facilitate the calculation of the $\mathrm{AB}$ magnitudes, and consequently the flux densities for each band, the $\mathrm{AB}$ offsets are provided in the fifth column.

\begin{tabular}{lcccc}
\hline \hline $\begin{array}{l}\text { Filter } \\
\text { name }\end{array}$ & $\begin{array}{c}\text { Effective } \\
\text { wavelength }(\AA)\end{array}$ & $\begin{array}{c}\text { Bandpass } \\
\text { width }(\AA)\end{array}$ & Magnitude & $\mathrm{AB}_{\text {off }}$ \\
\hline$U$ (ESO\#640) & 3711 & 166 & $23.54 \pm 0.30$ & 0.82 \\
$B$ & 4384 & 700 & $24.07 \pm 0.05$ & -0.13 \\
$V$ & 5368 & 527 & $23.80 \pm 0.06$ & 0.00 \\
STIS (50 CCD) & 6218 & 3538 & $23.76 \pm 0.10$ & 0.21 \\
$R$ & 6627 & 768 & $23.39 \pm 0.05$ & 0.26 \\
$I$ & 8007 & 778 & $22.79 \pm 0.05$ & 0.46 \\
$Z$ & 8940 & 993 & $22.46 \pm 0.10$ & 0.55 \\
$J_{\text {s }}$ (ISAAC) & 12499 & 958 & $22.27 \pm 0.10$ & 0.94 \\
$K_{\text {s }}$ (ISAAC) & 21638 & 1638 & $21.19 \pm 0.30$ & 1.87 \\
\hline
\end{tabular}

stars used for photometric calibration. Thus, for the groundbased optical and NIR pixel scales used in the present study ( $\geq 0$ ' $148 /$ pix, achieved with ISAAC), the GRB 000418 host galaxy can safely be assumed to be a point-source. Therefore, considering that the relative photometry is independent of the aperture radius, the $U B V R I Z J_{\mathrm{s}} K_{\mathrm{s}}$-band magnitudes shown in Table 2 are based on circular aperture (PHOT running under $\mathrm{IRAF}^{2}$ ) with no aperture corrections.

$B V R I Z$-band frames were taken with ALFOSC at the 2.5-m Nordic Optical Telescope (2.5 NOT). The ALFOSC detector is a $2048 \times 2048$ Thinned Loral CCD providing a pixel scale of 0 '. 189/pix. $U$-band observations were carried out with the 3.6-m ESO telescope (3.6 ESO) equipped with EFOSC2, covering a field of view $(\mathrm{FOV})$ of $5.5 \times 5.5$. These observations

\footnotetext{
${ }^{2}$ IRAF is distributed by the National Optical Astronomy Observatories, which is operated by the Association of Universities for Research in Astronomy, Inc. (AURA) under cooperative agreement with the National Science Foundation.
}

were carried out in $2 \times 2$ binning mode, providing a pixel scale of $0.314 /$ pix.

The optical data were reduced in a standard manner (overscan, bias subtraction, and division by a normalised flat field). The $U B V R I$-band calibration was based on the calibration given by Henden (2000). The Z-band calibration was carried out observing the spectro-photometric standard star Feige 66 (Oke 1990) with the $2.5 \mathrm{NOT}$ (+ALFOSC) at an airmass almost identical $(\Delta \sec (z)=0.03)$ to the GRB field. Figure 1 shows a deep $V$-band image of the GRB field taken with the 2.5 NOT(+ALFOSC).

The NIR ( $J_{\mathrm{s}}$ and $K_{\mathrm{s}}$-band) observations were acquired with the UT1 of the 8.2-m VLT (8.2 VLT) equipped with ISAAC, allowing us to cover a $2.5 \times 2.5$ FOV with a pixel scale of 0 ' $148 /$ pix. In Table 1 we provide the observing log of our optical and NIR observations. The calibration was based on observations of the standard stars S301-D ( $J_{\mathrm{s}}$ band) and S860-D ( $K_{\mathrm{s}}$ band; Persson et al. 1998). Due to the lack of 
$J_{\mathrm{S}}$-band calibration data for S301-D we assumed $J=J_{\mathrm{s}}$. Extinction coefficients of 0.06 and 0.07 mag per airmass (ESO Paranal) where assumed for $J_{\mathrm{s}}$ and $K_{\mathrm{s}}$, respectively. Given that the airmass difference between the GRB field and the standard was $\Delta \sec (z)=0.4$ in both $J_{\mathrm{s}}$ and $K_{\mathrm{s}}$, the introduced airmass correction is well below our measurement error on the magnitude of the host galaxy.

The photometric calibration has been tested performing photometry of the 2MASS star at RA, DEC $($ J2000) $=$ $12: 25: 14.47,20: 05: 49.8$ which is present on the ISAAC images taken for the host. Using the S301-D star we derived for this source $J_{\mathrm{s}}=15.51 \pm 0.01$ and $K_{\mathrm{s}}=14.87 \pm 0.01$. Both magnitudes agree with the ones given in the 2MASS Second Incremental Release Point Source Catalog $\left(J_{\mathrm{s}}=15.47 \pm 0.06\right.$ and $\left.K_{\mathrm{s}}=14.69 \pm 0.10\right)$.

\subsection{HST observations}

The HST observations were carried out under the ToO programme 8189 (Principal Investigator, Fruchter et al. 1999). The observations were performed at an epoch 299 days after the GRB (see Table 1) where the OA contribution is negligible. The observations were performed with STIS, which yields a gain of $1 \mathrm{e}^{-} / A D U$, a physical pixel scale of 0.05 and a FOV of $52^{\prime \prime} \times 52^{\prime \prime}$ (STScI 2000). The data were obtained with the $50 \mathrm{CCD}$ or $C L$ filter. The STIS reduction pipeline calstis of IRAF was used to calibrate the raw data. The raw images were reduced following normal procedures (bias and dark subtraction, and division by a normalised flat field). The individual images were combined using the drizzle task of IRAF (Fruchter \& Hook 2002). In the drizzling of the data the parameters pixfrac $=0.6$ and scale $=0.5$ were used. These values yield an output grid of $2 \mathrm{k} \times 2 \mathrm{k}$ pixels with a pixel scale of $0.0254 /$ pix. We performed aperture photometry of the host with a radius of 50 drizzled pixels. The count rate $(C$, measured in $A D U / \mathrm{s})$ was converted into the $C L$-band $\mathrm{AB}$ magnitude following: $m_{C L}^{\mathrm{AB}}=26.386-2.5 \log C(\mathrm{STScI} 2000)$.

In order to fit the observed Vega system magnitudes $(m)$ using a given template (either empirical or synthetic), the values of $m$ were converted into flux densities $\left(f_{v}\right)$ using the corresponding offsets to the $\mathrm{AB}$ magnitude system (Oke 1990). The $\mathrm{AB}$ offset is defined as $\mathrm{AB}_{\text {off }}=m_{\mathrm{AB}}-m$, where $m_{\mathrm{AB}}=$ $-2.5 \times \log f_{v}-48.60\left(f_{v}\right.$ measured in erg s $\left.\mathrm{s}^{-1} \mathrm{~cm}^{-2} \mathrm{~Hz}^{-1}\right)$ is the magnitude in the $\mathrm{AB}$ system. The $\mathrm{AB}$ offsets of our nine bands were derived convolving the Vega spectrum taken from the GISSEL98 (Bruzual \& Charlot 1993) library ( $\alpha$ Lyrae $m=0$ in all bands by definition) with the $U B V R I Z J_{\mathrm{S}} K_{\mathrm{S}} C L$-band filters and the corresponding CCD efficiency curves. Table 2 displays the $\mathrm{AB}$ offsets and effective wavelengths of the nine bands used to construct the SED. Prior to performing the SED fit, the derived $U B V R I Z J_{\mathrm{S}} K_{\mathrm{s}} C L$-band flux densities were dereddened of the Galactic extinction in the direction of the host $(E(B-V)=0.033$; Schlegel et al. 1998). The use of other Galactic extinction maps does not affect the final results of our analysis (Dickey \& Lockmann 1990 give $E(B-V)=0.057)$.

\section{Methodology}

\subsection{Construction of synthetic SED templates}

The applied synthetic SED fitting technique is the same as the one applied to the host galaxy of GRB 000210 (Gorosabel et al. 2003) and is based on Hyperz ${ }^{3}$ (Bolzonella et al. 2000). Eight synthetic spectral types were constructed representing Starburst galaxies (Stb), Ellipticals (E), Lenticulars (S0), Spirals (Sa, Sb, Sc and Sd) and Irregular galaxies (Im). The time evolution of the SFR for all galaxy types is represented by an exponential model, i.e. SFR $\propto \exp (-t / \tau)$, in which $\tau$ ranges from 0 (Stb) to $30 \mathrm{Gyr}(\mathrm{Sd})$. The SFR of Stb is modeled by an exponential decay in the limit $\tau \rightarrow 0$, in other words an instantaneous star burst. The early type galaxy spectra (E, S0) are represented by values of $\tau$ between 1 and 2 Gyr. The SFR of Im galaxies are represented by a constant $\operatorname{SFR}(\tau \rightarrow \infty)$.

The impact of the assumed Initial Mass Function (IMF) and the extinction law have been tested. Three IMFs were assumed, namely: Miller \& Scalo (1979; MiSc79), Scalo (1986; Sc86) and Salpeter (1955; Sp55). Christensen et al. (2003) show that the GRB 990712 host galaxy SED is better reproduced with a Sp55 IMF. However, the same method applied to GRB 000210 did not show any preference in the IMF (Gorosabel et al. 2003). According to Bolzonella et al. (2000) the Sp55 IMF produces an excess of bright blue stars producing an UV flux excess. On the other hand, the Sc86 IMF creates an excess in the number of solar mass stars, making the spectrum too red to reproduce the photometric points. Intensive photometric redshift studies have shown that the MiSc79 IMF is a good compromise between both tendencies (Bolzonella et al. 2002).

We have also tried to infer information on the host galaxy metallicity. The major effect of increasing the amount of metals contained in the host is to produce redder colours, hence altering the extinction determination. The impact of the metallicity has been tested comparing the results obtained when solar metallicity $\left(Z=Z_{\odot} \simeq 0.02\right.$; SOL $)$ and evolving metallicity templates are used. The metallicity evolution is based on the so-called closed-box model, which considers the ejection of heavy elements from each generation of stars assuming an instantaneous recycling of heavy elements. The two template groups (SOL and EVOL) have been combined with the above mentioned three IMFs, constituting 6 SED templates families (SOL-MiSc79, SOL-Sc86, SOL-Sp55, EVOLMiSc79, EVOL-Sc86 and EVOL-Sp55).

Furthermore, the effect of considering different extinction laws has been studied. For each of the 6 families 4 extinction laws have been taken into account for the determination of the photometric redshift and the physical conditions of the host, namely: Calzetti et al. (2000), Seaton (1979), Fitzpatrick (1986), and Prévot et al. (1984). The extinction laws will be abbreviated as, Cal00, Sea79, Fit86 and Pre84. Each of these extinction laws specifies the dependence of the extinction with frequency and is the result of different physical conditions in the interstellar space in the hosts. Thus, Sea79, Fit86 and Pre84, are representative of the Milky Way (MW), Large Magellanic Cloud (LMC) and the Small Magellanic Cloud (SMC)

\footnotetext{
${ }^{3}$ http://webast.ast.obs-mip.fr/hyperz/
} 

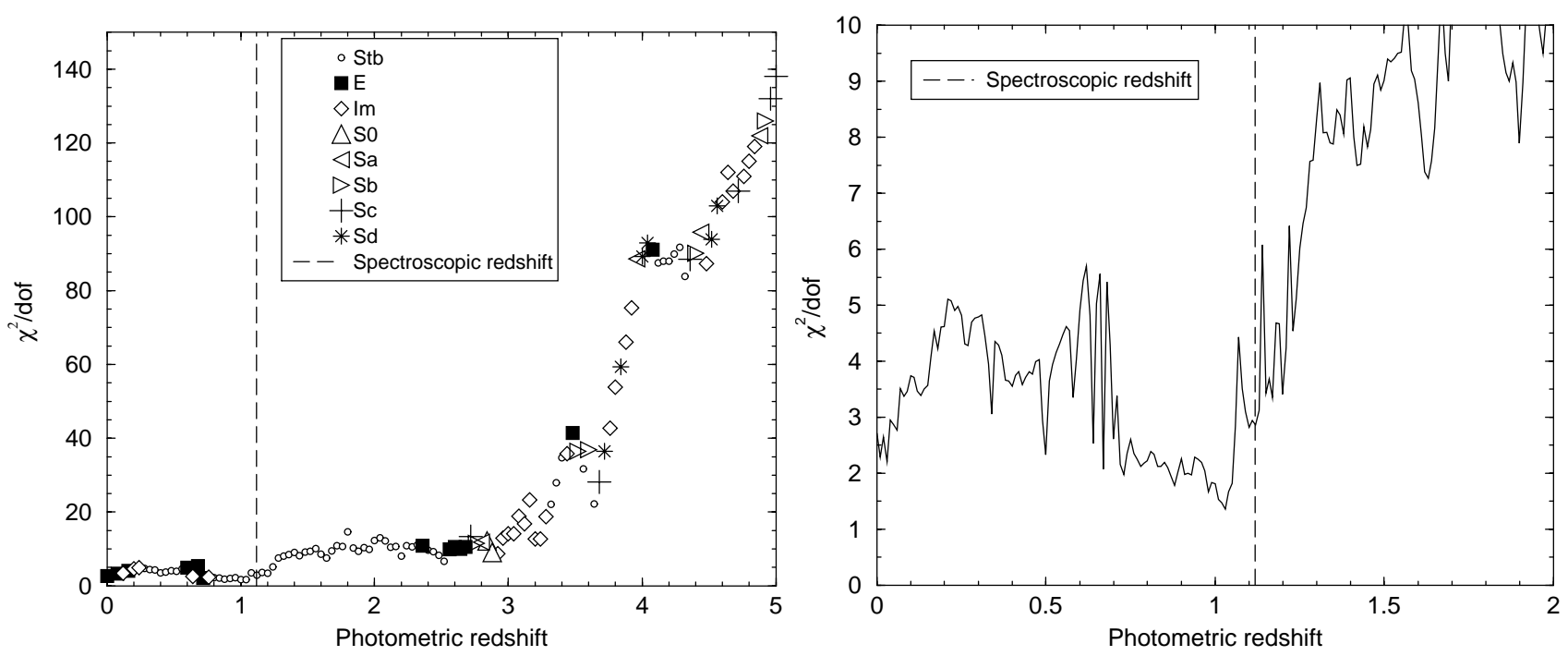

Fig. 2. Left panel: The global mapping of the fitted SED $\chi^{2} /$ d.o.f. when the redshifts of the templates are varied in a broad range $(0<z<5)$. The dotted vertical line indicates the spectroscopic redshift of the host galaxy $(z=1.118)$. The different symbols indicated the best fitted template type for each redshift. As it is shown, the spectroscopic redshift is consistent with the broad minimum $(0.65<z<1.20)$ centred at $z=1.029$. This minimum is governed by Stb galaxies. Other local minima (for instance the one at $z \sim 0$ achieved with $\mathrm{E}$ and Im galaxies) show larger values of $\chi^{2} /$ d.o.f. Right panel: The plot shows a blow up around the absolute minimum with no distinction among templates types. The evolution of $\chi^{2} /$ d.o.f. rejects the photometric redshifts not located at $0.65<z<1.2$, specially the ones at $z>1.5$ where $\chi^{2} /$ d.o.f. $>9$. General: The plot assumed a Sc86 IMF, a Sea79 extinction law, and Solar metallicity (SOL-Sc86-Sea79 subfamily of templates).

extinction laws, respectively. The Cal00 extinction law is suitable for starburst regions.

Due to computational limitations, the fitting strategy was divided in two steps. As a first stage, the redshifts and the extinctions of the templates were varied in a broad range $\left(0<z<5,0<A_{V}<5\right.$; see left panel of Fig. 2) in order to map globally the $\chi^{2} /$ d.o.f. evolution. The used steps were $\Delta z=0.05$ and $\Delta A_{V}=0.05$, respectively. Once a deep broad minimum of $\chi^{2} /$ d.o.f. was localized around $z \sim 1$, the accurate fit parameters (displayed in Table 3) were determined with a thinner grid of SED templates ( $\Delta z=0.01, \Delta A_{V}=0.03$; see right panel of Fig. 2). In this second stage, $z$ and $A_{V}$ were ranged around the $\chi^{2} /$ d.o.f. minimum $\left(0<z<2\right.$ and $\left.0<A_{V}<3\right)$.

\subsection{Comparison with empirical SED templates}

We have complemented the analysis carried out with the synthetic templates by using the 12 empirical templates by Kinney et al. (1996; hereafter K96). Those templates reproduce 6 SEDs of quiescent (Bulge(B), E, S0, Sa, Sb and Sc) and 6 of starburst galaxies. The templates of the quiescent galaxies are built according to morphological type, and the starburst galaxies according to colour excess (Stb1, Stb2, Stb3, Stb4, Stb5 and Stb6; see K96). The starburst galaxy SEDs are represented by a grid of templates constructed increasing $E(B-V)$ from 0.05 to 0.65 with a step of 0.1 (see Col. 3 of Table 4 ).

The early-type empirical templates (B, E and S0) have the reddest colours (dominated by evolved stellar populations) with a large flux density increase (in the $F_{\lambda}$ representation) from the UV to the optical wavelengths, specially at the $4000 \AA$ break. The Sa and Sb galaxies tend to be slightly bluer. For all the early and mid type templates (B, E, S0, Sa and Sb) the flux density $F_{\lambda}$ increases with lambda. The Sc template is in the transition towards the blue starburst galaxies and can be approximated by a flat spectrum. The starburst templates show spectra that rise toward shorter wavelengths. They become increasingly bluer, as $E(B-V)$ decreases progressively from Stb6 to Stb1.

The wavelength coverage of the K96 templates (1000$10000 \AA$ ) have been extended to the near-IR by means of Bruzual \& Charlot (1993) spectra with parameters selected to match the K96 spectra at $z=0$. In any case, for the redshift of the host $(z=1.118)$ the extrapolation is not crucial because only the $K_{\mathrm{S}}$ band falls beyond the restframe IR limit (10000 $\left.\mathrm{A}\right)$ of the K96 templates.

The fits have been performed fixing the redshift of the host at the spectroscopic redshift. For the quiescent galaxy templates the extinction law given by Sea79 has been assumed, leaving $E(B-V)$ as a free parameter. For the starburst galaxies the used extinction law was the one of Cal00, fixing $E(B-V)$ according to the definition of the corresponding template. Table 4 shows the results obtained for each template.

\section{Results}

\subsection{Underlying morphological structure}

We have built a model of the galaxy based on ellipses of constant surface brightness. Once the model has been constructed it has been subtracted from the observed surface brightness in order to detect residuals which could reveal the presence of underlying structures (like dust lanes, spiral arms or disks). This method was already successfully applied to the HST/STIS data taken for the GRB 980703 host galaxy, suggesting subjacent structures or/and an irregular morphology (Holland et al. 2001). Similar techniques also found significant structure in the 
Table 3. The table displays the parameters of the best host galaxy SED fit when several IMFs and extinction laws, indicated in the first and second columns, are adopted. The rest of the columns display the inferred parameters under the assumed IMF and extinction law. The third column provides the confidence of the best fit (given by $\chi^{2} /$ d.o.f., being d.o.f. $=8$ ). The derived photometric redshift is displayed in the fourth column (and the corresponding 68\% and 99\% percentile errors). In the fifth and sixth columns the template family of the best fitted SED and the age of the stellar population are given. The seventh column displays the derived value of the host galaxy extinction $A_{V}$. The eighth column displays the derived rest frame absolute $B$-band magnitude, $M_{B}$. The ninth column gives the Luminosity of the host in units of $L^{\star}$ (Schechter 1976). The last column displays $S_{F} R_{U V}$ derived from the $2800 \AA$ flux once it is corrected for the corresponding reddening (see Sect. 4.2 .4 for a detailed discussion on the $\mathrm{SFR}_{\mathrm{UV}}$ estimation). The table is divided in three sub-tables. The upper sub-table displays the derived parameters when a constant solar metallicity is assumed. The middle one assumes a metallicity evolving in time. The lower sub-table displays the results when no extinction is assumed.

\begin{tabular}{|c|c|c|c|c|c|c|c|c|c|}
\hline IMF & Extinction law & $\begin{array}{c}\chi^{2} / \text { d.o.f. } \\
\text { (d.o.f. }=8)\end{array}$ & $\begin{array}{c}\text { Photometric redshift } \\
z_{-p 68 \%, p 99 \%}^{+p 68 \%, p 99 \%}\end{array}$ & Template & $\begin{array}{l}\text { Age } \\
(\mathrm{Gyr})\end{array}$ & $A_{V}$ & $M_{B}$ & $L / L^{\star}$ & $\begin{array}{c}\mathrm{SFR}_{\mathrm{UV}} \\
M_{\odot} \mathrm{yr}^{-1}\end{array}$ \\
\hline \multicolumn{10}{|c|}{ Constant metallicity $\left(Z=Z_{\odot} \simeq 0.02\right)$} \\
\hline Sp55 & $\mathrm{Cal00}$ & 0.724 & $1.005_{-0.004,0.281}^{+0.022,057}$ & Stb & 0.004 & 1.38 & -20.41 & 0.84 & $60.4 \pm 27.3$ \\
\hline MiSc79 & $\mathrm{Cal} 00$ & 0.835 & $1.004_{-0.010 .0 .254}^{+0.004,0.055}$ & Stb & 0.004 & 1.47 & -20.38 & 0.82 & $70.1 \pm 33.7$ \\
\hline Sc86 & $\mathrm{Cal} 00$ & 0.739 & $1.004_{-0.006,0.057}^{+0.027,0.057}$ & Stb & 0.004 & 1.35 & -20.40 & 0.83 & $57.5 \pm 25.4$ \\
\hline Sp55 & Pre84 & 0.723 & $1.001_{-0.011,0.017}^{+0.004,0.052}$ & $\mathrm{E}$ & 0.181 & 0.42 & -20.39 & 0.82 & $14.2 \pm 1.0$ \\
\hline MiSc79 & Pre84 & 0.750 & $1.001_{-0.010 .0 .052}^{+0.004,0.052}$ & $\operatorname{Im}$ & 0.128 & 0.39 & -20.39 & 0.82 & $13.4 \pm 0.9$ \\
\hline Sc86 & Pre84 & 0.673 & $1.020_{-0.004,0.0075}^{+0.009,0.033}$ & $\mathrm{E}$ & 0.128 & 0.36 & -20.44 & 0.86 & $12.6 \pm 0.8$ \\
\hline Sp55 & Fit86 & 1.349 & $1.020_{-0.005,0.0068}^{+0.012,0.032}$ & Stb & 0.053 & 0.18 & -20.43 & 0.86 & $8.5 \pm 0.3$ \\
\hline MiSc79 & Fit86 & 1.395 & $1.017_{-0.003,0.069}^{+0.015,0.035}$ & Stb & 0.053 & 0.18 & -20.42 & 0.85 & $8.5 \pm 0.3$ \\
\hline Sc86 & Fit86 & 1.319 & $1.029_{-0.014,0.0069}^{+0.005,0.024}$ & Stb & 0.053 & 0.12 & -20.46 & 0.88 & $7.6 \pm 0.3$ \\
\hline Sp55 & Sea79 & 1.431 & $1.031_{-0.018,0.0079}^{+0.003,0.022}$ & Stb & 0.064 & 0.12 & -20.45 & 0.87 & $7.6 \pm 0.3$ \\
\hline MiSc79 & Sea79 & 1.472 & $1.019_{-0.006,0.072}^{+0.014 .0 .033}$ & Stb & 0.053 & 0.15 & -20.43 & 0.86 & $8.1 \pm 0.3$ \\
\hline Sc86 & Sea79 & 1.358 & $\begin{array}{r}1.029_{-0.012,0.069}^{+0.005,0.025} \\
\end{array}$ & Stb & 0.053 & 0.12 & -20.46 & 0.88 & $7.6 \pm 0.3$ \\
\hline \multicolumn{10}{|c|}{ Evolving metallicity } \\
\hline Sp55 & $\mathrm{Cal00}$ & 0.724 & $1.005_{-0.022,0.251}^{+0.022,0.055}$ & Stb & 0.004 & 1.38 & -20.41 & 0.84 & $60.4 \pm 27.3$ \\
\hline MiSc79 & Cal00 & 0.835 & $1.004_{-0.022,0.253}^{+0.024,0.055}$ & Stb & 0.004 & 1.47 & -20.38 & 0.82 & $70.1 \pm 33.7$ \\
\hline Sc86 & Cal00 & 0.739 & $1.004_{-0.006,0.250}^{+0.027,0.057}$ & Stb & 0.004 & 1.38 & -20.40 & 0.83 & $60.4 \pm 27.3$ \\
\hline Sp55 & Pre84 & 0.668 & $1.019_{-0.009,0.323}^{+0.013,0.040}$ & $\mathrm{E}$ & 0.181 & 0.36 & -20.46 & 0.88 & $12.6 \pm 0.8$ \\
\hline MiSc79 & Pre84 & 0.596 & $1.017_{-0.005,0.320}^{-0.0015,0.043}$ & $\mathrm{E}$ & 0.128 & 0.36 & -20.46 & 0.88 & $12.6 \pm 0.8$ \\
\hline Sc86 & Pre84 & 0.507 & $1.016_{-0.005,0.061}^{-0.0016,0.020}$ & $\mathrm{E}$ & 0.128 & 0.33 & -20.46 & 0.88 & $11.9 \pm 0.7$ \\
\hline Sp55 & Fit86 & 1.349 & $1.020_{-0.005,0.068}^{+0.012,0.032}$ & Stb & 0.053 & 0.18 & -20.43 & 0.86 & $8.5 \pm 0.3$ \\
\hline MiSc79 & Fit86 & 1.395 & $1.013_{-0.004,0.0069}^{+0.0156}$ & Stb & 0.053 & 0.18 & -20.42 & 0.85 & $8.5 \pm 0.3$ \\
\hline Sc86 & Fit86 & 1.319 & $1.029_{-0.014,0.0069}^{+0.015,0044}$ & Stb & 0.053 & 0.12 & -20.46 & 0.88 & $7.6 \pm 0.3$ \\
\hline Sp55 & Sea79 & 1.431 & $1.031_{-0.018,0.079}^{+0.003,0.022}$ & Stb & 0.064 & 0.12 & -20.45 & 0.87 & $7.6 \pm 0.3$ \\
\hline MiSc79 & Sea79 & 1.472 & $1.019_{-0.006,0.0072}^{+0.003,0.014}$ & Stb & 0.053 & 0.15 & -20.43 & 0.86 & $8.1 \pm 0.3$ \\
\hline Sc86 & Sea79 & 1.358 & $\begin{array}{l}1.029_{-0.012,0.069}^{+0.005,0.025} \\
\end{array}$ & Stb & 0.053 & 0.12 & -20.46 & 0.88 & $7.6 \pm 0.3$ \\
\hline \multicolumn{10}{|c|}{ No extinction } \\
\hline Sp55 & - & 1.559 & $1.011_{-0.002,0.007}^{+0.002,0.040}$ & Stb & 0.091 & 0.0 & -20.42 & 0.85 & $6.2 \pm 0.2$ \\
\hline MiSc79 & - & 1.659 & $1.011_{-0.002,2.0009}^{+0.0002,0040}$ & Stb & 0.091 & 0.0 & -20.41 & 0.84 & $6.2 \pm 0.2$ \\
\hline Sc86 & - & 1.646 & $1.029_{-0.008,0.0034}^{+0.0033}$ & Stb & 0.064 & 0.0 & -20.47 & 0.89 & $6.2 \pm 0.2$ \\
\hline
\end{tabular}

inner part of AGNs, supporting the scenario of central supermassive black holes (Xilouris \& Papadakis 2002).

The mentioned ellipses of constant surface brightness where obtained using the isophote package in IRAF. The surface photometry is analysed by the task ellipse, which allows the construction of the galaxy model by means of the bmodel IRAF task. The determination of the residual image was performed in two steps. First the constructed model was subtracted from the observed surface brightness providing a preliminary residual. This residual was used to make a mask that excluded other nearby objects from the model. If the pixel value in the residual image deviates by more than $2 \sigma$ from the input image, the pixel is flagged and not used in a new run of ellipse and bmodel.

The left panel of Fig. 3 shows the coadded STIS 50 CCDband image of the host galaxy. The right panel shows the subtracted image where only a few pixels are more than $2 \sigma$ above the background noise level. We do not detect any underlying 

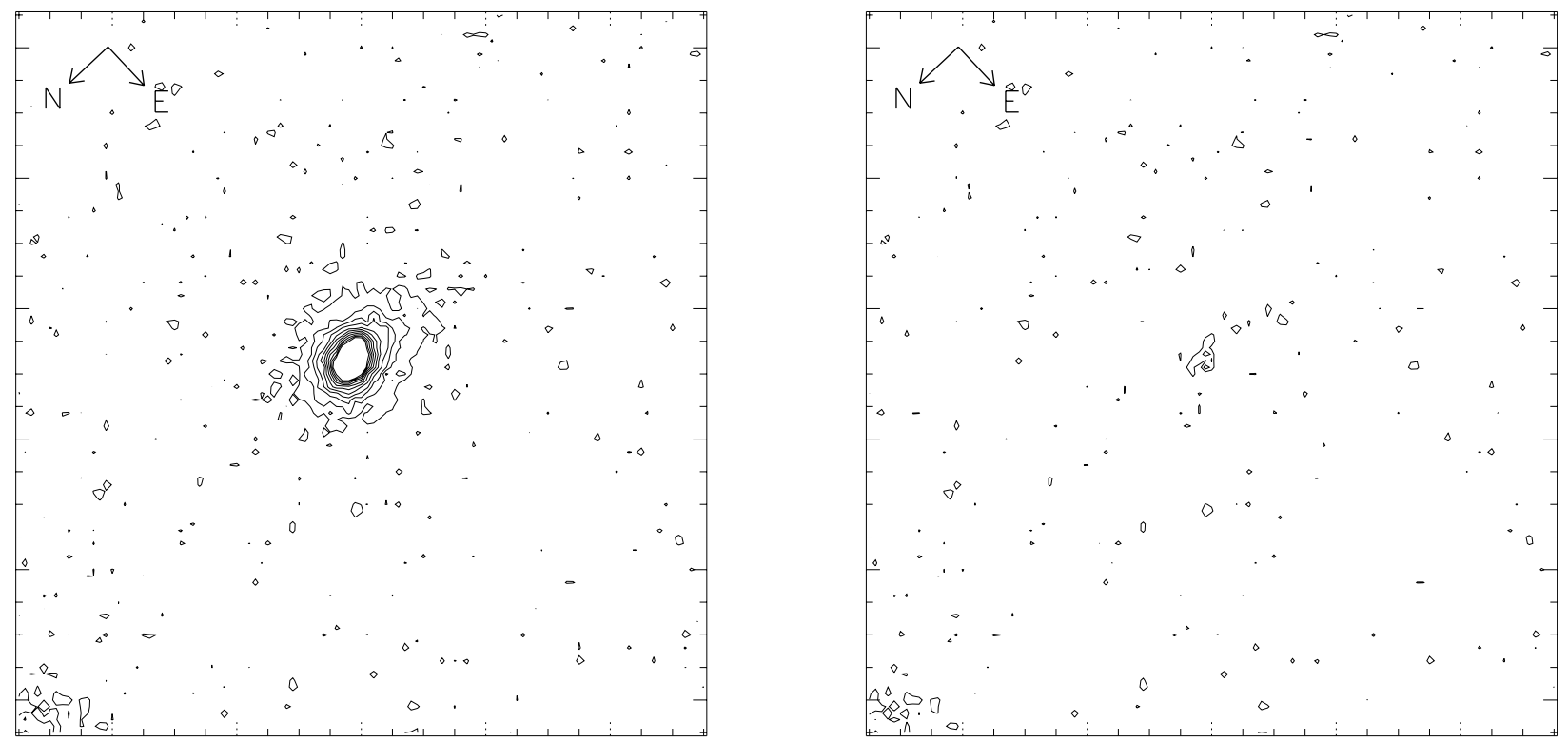

Fig. 3. The left panel shows a contour plot of the GRB 000418 host galaxy seen with STIS (CL-band filter). The observations were carried out 299 days after the gamma-ray event, so no contribution from the OA is expected. The right panel contours show the residuals of the host when the host galaxy surface brightness model is subtracted (see Sect. 4.1 for further information). The contours of both figures scale linearly with the detection confidence level (from $2 \sigma$ to $30 \sigma$, with a step of $3 \sigma$ ). As can be seen in the right plot, the residuals consist of a few pixels $2 \sigma$ above the background. They are concentrated in the host nucleus, where the PSF of STIS might affect the surface photometry. Thus, we conclude that (perhaps with the exception of the central region within a radius of 0 ' $^{\prime} 075$ ) there are no reliable underlying structures associated to the host galaxy (like dust lanes, spiral arms or disks) above the detection threshold of our STIS images. The FOV is 2 '. $8 \times 22^{\prime \prime} 8$. The arrows indicate the orientation of the images.

structure further than $0.075(0.66 \mathrm{kpc}$ at $z=1.118)$ from the center of the host.

A detailed morphological study of the GRB 000418 host radial profile (taking into account the effects of the HST/STIS PSF) is beyond the scope of the present study. Such analysis can be found in Vreeswijk (2002), who report that both a de Vaucouleurs and an exponential profile provide reasonable fit to the data.

\subsection{Information derived from the SED fits}

Figure 4 shows the SED of the GRB 000418 host galaxy. Table 3 displays the result of our SED fits based on synthetic templates. Table 4 displays the complementary results obtained when the empirical K96 templates are fitted.

The discussion on the impact of the mentioned SED fitting methods and the corresponding assumed parameters is split as follows.

\subsubsection{Galaxy type}

According to Table 3 most of the IMF and extinction combinations favour a starburst galaxy. The exception occurs when a Pre84 extinction law is assumed. In most of those cases a young Elliptical galaxy template is the best fitted SED, with a stellar age $0.128-0.181$ Gyr. In one case (SOL-MiSc79) the Pre84 extinction law is consistent with a young $(0.128 \mathrm{Gyr})$ Im galaxy template.

In the first stages of the SED evolution, when the galaxy is dominated by a young blue stellar population (typically
Table 4. Results obtained based on the empirical templates by Kinney et al. (1996). The templates have been ordered according to their colour, bluer on the top (Stb1) and redder on the bottom (B). As it can be seen there is a clear correlation between the goodness of the fit and the colour of the template: the bluer the colour the lower $\chi^{2} /$ d.o.f. While Stb type galaxies (Stb1, Stb2, Stb3, Stb4, Stb5 and Stb6) have their extinction fixed by the template definition, for the quiescent ones (Sc, Sb, Sa, S0, E and B) the extinction can take any arbitrary value. In all these early and mid type cases the blue SED predicted by our photometric data is reproduced more closely by the bluest fit possible. Introduction of dust reddening for Sc, Sb, Sa, S0, E and B would just worsen the fit. This is why in the last column of the table $E(B-V)=0.0$ for the quiescent galaxies.

\begin{tabular}{lcc}
\hline \hline $\begin{array}{l}\text { Galaxy } \\
\text { Template }\end{array}$ & $\begin{array}{c}\chi^{2} / \text { d.o.f. } \\
\text { (d.o.f. }=8)\end{array}$ & $E(B-V)$ \\
\hline Stb1 & 5.92 & $0.05 \pm 0.05^{\dagger}$ \\
Stb2 & 4.81 & $0.15 \pm 0.05^{\dagger}$ \\
Stb3 & 23.71 & $0.30 \pm 0.05^{\dagger}$ \\
Stb4 & 37.00 & $0.45 \pm 0.05^{\dagger}$ \\
Stb5 & 48.29 & $0.55 \pm 0.05^{\dagger}$ \\
Stb6 & 70.51 & $0.65 \pm 0.05^{\dagger}$ \\
Sc & 66.23 & 0.0 \\
Sb & 206.01 & 0.0 \\
Sa & 225.86 & 0.0 \\
S0 & 249.38 & 0.0 \\
E & 255.08 & 0.0 \\
B & 255.65 & 0.0 \\
\hline
\end{tabular}

$\dagger$ Extinction fixed by the template definition. 


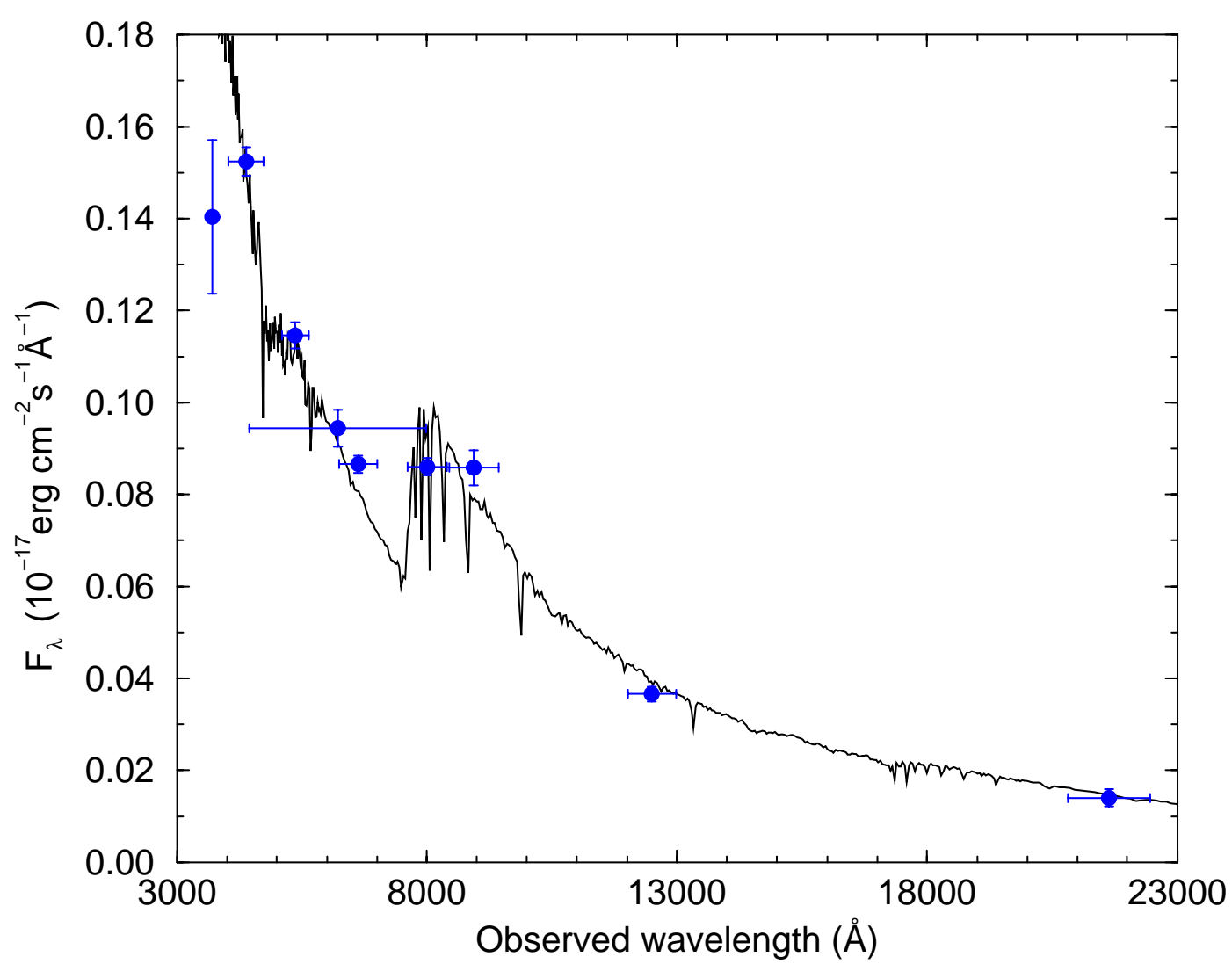

Fig. 4. The points show the measured flux in the $U B V R I Z J_{\mathrm{s}} K_{\mathrm{s}} C L$ bands for the GRB 000418 host galaxy, once the Galactic dereddening is introduced $\left(E(B-V)=0.033\right.$, Schlegel et al. 1998). The solid curve represent the best SED fitted to the photometric points $\left(\chi^{2} /\right.$ d.o.f. $\left.=1.358\right)$, corresponding to a starburst synthetic template at a redshift of $z=1.029$. The plots assumes a Sc86 IMF. The derived value of the starburst age corresponds to $0.053 \mathrm{Gyr}$. The extinction law used to construct the plot is given by Sea79. The fit is consistent with a low global extinction in the host $\left(A_{V}^{\text {global }} \sim 0.12\right)$. The SED shows an appreciable $\sim 4000 \times(1+z) \AA$ break, approximately at the $Z$-band filter wavelength.

younger than $0.1 \mathrm{Gyr}$ ) all the synthetic templates resemble each other. The templates start to differentiate at ages $>0.1 \mathrm{Gyr}$, when the $4000 \AA$ break becomes progressively prominent. Thus, the ages inferred for the $\mathrm{E}$ and Im templates are close to that evolutionary transition present at $\sim 0.1 \mathrm{Gyr}$, when their SEDs are still similar to starbursts. The low age derived for the $\mathrm{E}$ and Im templates is just an indication that undergoing active stellar formation is present in the host, independently of the type assigned to each template (the difference among the young templates is more due to a template name definition than a real physical reason). So, only based on the analysis of synthetic templates we can not completely rule out that the host SED is not a young $\mathrm{E}$ or an Im galaxy. However, even for these few cases (which represent $22 \%$ of the SED solutions displayed in Table 3), we can claim that the galaxy harbours a prominent blue stellar formation activity. Therefore, it is clear that the SED is only reproducible with a young synthetic stellar population and it is incompatible with an evolved population.

The starburst scenario is also supported by the results obtained from fitting the K96 observational templates. As it is shown in Table 4, by far the smallest values of $\chi^{2} /$ d.o.f. are obtained with blue, low extinction, starburst templates (Stb2 and a Stb1). The rest of the empirical templates, especially the early type galaxies (S0, E and B), show a high degree of discrepancy with the photometric points $\left(\chi^{2} /\right.$ d.o.f. $>23.71$, see Table 4$)$.
Therefore, combining the morphological information and the synthetic and empirical SED fits we conclude that the GRB 000418 host galaxy SED is best reproduced by a Stb template. This agrees with the independent result reported by Bloom et al. (2003) who based on the relative intensities of the [O II], He I, [Ne III] and $\mathrm{H} \gamma$ lines, conclude that the host is a starburst galaxy rather than a LINER or a Seyfert 2 galaxy.

The lack of underlying peripheric bright knots of star formation (see Sect. 4.1) supports a model with one dominant nuclear starburst. This fact makes our photometric points a suitable input for Hyperz, as multiple contemporaneous episodes of star formation can not be fitted with this code.

\subsubsection{Metallicity}

In the upper sub-table of Table 3 we show the results obtained when solar metallicity is assumed for the host galaxy. In the middle sub-table the results are displayed when the metallicity is not fixed. In such case the stars eject heavy elements to the environment, enriching the ISM where new generation of stars are continuously born. The effect of the ISM enrichment is expected to be maximum for Im galaxies (where the SFR is constant) and negligible for instantaneous starbursts, where all the stars are modeled to be formed at the same epoch (instantaneous SFR idealised by a delta function). 
As it is shown the results of both sub-tables are basically the same. Even if the metallicity is left as a free parameter the Stb template is the one providing the most satisfactory fits. Thus, we conclude that the metallicity of the host is consistent with Solar metallicity, but that the metallicity is not strongly constrained by our analysis.

\subsubsection{Extinction}

According to the empirical SED templates, the blue SED of the host can only be roughly reproduced with low extinction starburst galaxies (templates Stb1 and Stb2). As it can be seen in Table 4 there is a clear correlation between the goodness of the fit and the colour of the template: the bluer the colour the lower $\chi^{2} /$ d.o.f. To translate the value of $E(B-V)$ to $A_{V}^{\text {global }}$ we adopt the value of $R_{V}=A_{V}^{\text {global }} / E(B-V)=4.05 \pm 0.80$ proposed for starburst galaxies (Calzetti et al. 2000). The best fit is achieved with a Stb2 template, which shows by definition a fixed colour excess $E(B-V)=0.15 \pm 0.05\left(A_{V}^{\text {global }}=0.61 \pm\right.$ 0.24 following Cal00).

The host galaxy extinction $A_{V}^{\text {global }}$ derived from the synthetic SEDs range from 0.12 to $1.47 \mathrm{mag}$ (see Table 3 ). The derived $A_{V}^{\text {global }}$ values are mostly dependent on the assumed extinction law, and almost independent of the IMF and metallicity. The Cal00 extinction law predicts the existence of a very blue, young (age $\sim 4 \mathrm{Myr}$ ) and extincted stellar population $\left(A_{V}^{\text {global }} \sim 1.4\right)$. However, the inferred high $A_{V}^{\text {global }}$ is not supported by the empirical template fits, so this solution seems quite unlikely and for the further discussion it will only be considered as a secondary scenario.

For illustrative purposes, we have included in the lower sub-table of Table 3 the results obtained when we impose $A_{V}^{\text {global }}=0$. As it is shown these fits are still satisfactory $\left(\chi^{2} /\right.$ d.o.f. $\left.\sim 1.6\right)$ and the photometric redshift is very close to the spectroscopic one $(z \sim 1.02)$, implying that the assumption of no extinction is not in strong conflict with the data.

In conclusion, the synthetic and empirical templates yield similar results; the fits to the GRB 000418 host galaxy SED lead to a low/moderate $A_{V}^{\text {global }}$ ranging from 0.12 (e.g. Sc86Sea79 synthetic SED) to $0.61 \mathrm{mag}$ (Stb2 empirical template). In the following we will assume $A_{V}^{\text {global }}=0.36 \pm 0.25$. This value is consistent with the extinction along the line-of-sight to the OA $\left(A_{V}^{\text {local }}=0.65 \pm 0.25\right)$. Given that $A_{V}^{\text {local }}$ and $A_{V}^{\text {global }}$ are not very different we conclude that the GRB apparently did not occur in a region with more than average extinction.

\subsubsection{Star Formation Rate}

The UV continuum emission is dominated by bright, shortlived, main-sequence $\mathrm{O}$ and $\mathrm{B}$ stars. According to Kennicutt (1998) the rest-frame $2800 \AA$ flux is directly proportional to the star-formation rate in the host galaxy. Therefore, the SFR can be estimated from this part of the spectrum.

When this diagnostic method is applied to starburst galaxies it is affected by two main uncertainties. The first one is the sensitivity of the estimator to extinction, which has a strong impact on the UV region. The young massive stars can be located in regions where the $A_{V}^{\text {local }}$ properties differ substantially from the average host extinction (derived from the SED fitting), so a proper UV correction is difficult to obtain. The second uncertainty comes from the fact that the expression given by Kennicutt (1998) relating the $\mathrm{SFR}_{\mathrm{UV}}$ and the UV luminosity $\left(L_{\mathrm{UV}}\right)$ is only strictly valid for galaxies with continuous star formation over time scales of $10^{8} \mathrm{yrs}$ or longer. The $\mathrm{SFR}_{\mathrm{UV}} / L_{\mathrm{UV}}$ ratio is significantly lower for younger populations such as starburst galaxies. Hence, these two systematic uncertainties have opposite effects on the derived SFR.

At $z=1.118$ the rest-frame $2800 \AA$ corresponds to $5930 \AA$, so it is bracketed between our $V$ and $R$ bands. The flux at $5930 \AA$ has been determined by fitting a power law spectrum to the $V$ and $R$-band photometric points (once corrected for Galactic extinction) and then interpolating it at $5930 \AA$. Finally, the interpolated flux has been dereddened according to the corresponding extinction law and the $A_{V}^{\text {global }}$ value inferred with the synthetic SED fitting (see Col. 7 of Table 3). The resulting SFRs can be seen in Table 3 .

As it is shown, the SFR $_{\mathrm{UV}}$ estimates displayed in Table 3 (once the unlikely SED solutions have been discarded) are $\sim 7$ times lower than the $\mathrm{SFR}_{[\mathrm{OII}}$ estimates based on the [O II] line flux, $L[\mathrm{O}$ II] (Bloom et al. 2003). The main reason for this disagreement is the high $\mathrm{SFR}_{[\mathrm{O} \text { II }} / L\left[\mathrm{O}\right.$ II] ratio $\left(5 \times 10^{-41}\right.$, given by Kennicutt 1992) assumed by Bloom et al. (2003). If the more updated $\mathrm{SFR}_{[\mathrm{O}}{ }_{\mathrm{II}} / L[\mathrm{O} \mathrm{II}]$ ratio $\left(1.4 \times 10^{-41}\right)$ reported by Kennicutt (1998; the reference used in our study to estimate $\mathrm{SFR}_{\mathrm{UV}}$ ) is applied, then the $L[\mathrm{O}$ II $]$ measured by Bloom et al. (2003) corresponds to $\left.\mathrm{SFR}_{[\mathrm{O}}{ }_{\mathrm{II}}\right]=15.4 M_{\odot} \mathrm{yr}^{-1}$ (with a systematic error of $\sim 30 \%$ ), close to our $\mathrm{SFR}_{\mathrm{UV}}$ estimate.

A second order parameter that could explain the factor of two still present between both SFR estimates might be the naive scenario we assumed for the local extinction correction. In principle, if the local extinction estimation was perfect, the UV and the [O II] diagnostic methods should yield same results. However, if (part or most of) the massive star population responsible of the UV radiation is embedded in extincted regions, then the $A_{V}^{\text {global }}=A_{V}^{\text {local }}$ approximation (used to derive the $S_{F R}$ values displayed in Table 3 ) would underestimate the UV flux.

Additionally, the reddening correction for $\mathrm{SFR}_{[\mathrm{O} \text { II }}$ has to be carried out at $\mathrm{H}_{\alpha}(6563 \AA)$ and not at [O II] (3727 $\AA$ ), given the manner the [O II] fluxes were calibrated (Kennicutt 1992, 1998). This makes the extinction correction for the $\mathrm{SFR}_{\mathrm{UV}} / \mathrm{SFR}_{[\mathrm{O} \text { II }}$ ratio even higher.

Considering the above mentioned uncertainties and limitations, intrinsic to both SFR diagnostic techniques, we conclude that the $\mathrm{SFR}_{\mathrm{UV}}$ based on the our $U B V R I Z J_{\mathrm{S}} K_{\mathrm{S}} C L$-band SED is compatible with the $\mathrm{SFR}_{[\mathrm{O} \text { II] }}$ estimated by Bloom et al. (2003).

\subsubsection{Stellar population age}

Information on the stellar population age can be inferred from the strength of the $4000 \AA$ jump by means of synthetic templates. The age of the derived Stb synthetic SEDs (the rest of synthetic templates have been considered much more unlikely; 
see Sects. 4.2.1 and 4.2.3) range from 0.053 to 0.064 Gyr. An age of $0.059 \pm 0.006 \mathrm{Gyr}$ (the mean of value of 0.053 and 0.064 ) corresponds (basically independent of the metallicity) to the lifetime of a star with $7 \pm 1 M_{\odot}$ (see Table 14 of Portinari et al. 1998).

\subsubsection{Photometric redshift}

Given that the spectroscopic redshift is known, the photometric redshift is only used to check the internal consistency of the fitted SEDs. A systematic comparison performed between spectroscopic and photometric redshifts inferred with Hyperz for a sample of $10 \mathrm{GRB}$ hosts shows a typical redshift dispersion $\sim 0.1$ (Christensen et al. 2002). Thus, we conclude that the synthetic SED fits reproduce reasonably well the spectroscopic redshift of our host galaxy (see fourth column of Table 3).

We have checked the possible impact that the poorly determined $U$-band magnitude (error $0.3 \mathrm{mag}$ ) might have in the determination of the photometric redshift. Thus, we have repeated all the fits displayed in Table 3 excluding the $U$-band host magnitude. The derived photometric redshifts differ less than $2 \%$ (achieved for the SOL-MiSc79-Fit86 subfamily of templates) from the ones obtained with the entire $U B V R I Z J_{\mathrm{S}} K_{\mathrm{s}} C L$-band SED. This small variation is the result of the weighted calculation of $\chi^{2} /$ d.o.f., which weights each band according to the square of its corresponding photometric error inverses (see Hyperz manual, Bolzonella et al. 2002). In the same manner the impact of the $U$-band magnitude on rest of the inferred variables (galaxy type, metallicity, age, template, $A_{V}^{\text {global }}$ ) is also negligible for the further discussion (the maximum impact corresponds to a variation of one $A_{V}^{\text {global }}$ grid step, $0.03 \mathrm{mag}$ ).

The reliability of our empirical templates fits have been also tested. Leaving the redshift as a free parameter, (and filtering the spurious local $\chi^{2} /$ d.o.f. minimum frequently found at $z=0$ ), only the Stb2 template yields a reasonable photometric redshift $(z=1.272$, consistent within the expected redshift dispersion of $\Delta z \sim 0.1$ ). The rest of templates, specially the early and mid types, give redshifts inconsistent with the spectroscopic one. This fact supports that the Stb2 empirical template (see Sect. 4.2.3) is the optimum one to reproduce our data.

\subsubsection{Luminosity of the host galaxy}

The absolute $B$-band magnitude of the host at $z=1.118$ is $M_{B}=-20.6 \pm 0.1$. Lilly et al. (1995) show that $M_{B}^{\star}$ depends on the colour and the redshift of the galaxy. This luminosity evolution is specially relevant for blue galaxies at $z \sim 1$ (like the GRB 000418 host galaxy), where $M_{B}^{\star}$ ranges from -21.22 to -22.93 (rescaling the $M_{B}^{\star}$ values of Lilly et al. (1995) to our cosmology). Although the $M_{B}^{\star}$ value of blue galaxies is very uncertain, the trivariate luminosity function (LF) of Lilly et al. (1995) suggests that the value of $M_{B}^{\star}$ of blue galaxies is $<-20.6$. Therefore, we conclude that the host is likely a subluminous galaxy.

\section{Discussion: Fitting all the pieces of the puzzle}

Several characteristics of the GRB 000418 host galaxy are difficult to reconcile, in particular: $i$ ) a high reddening is expected for sub-mm luminous galaxies (see Le Floc'h et al. 2003). However, we find that GRB 000418 occurred in a blue host galaxy with a low/moderate extinction; ii) $A_{V}^{\text {local }} / A_{V}^{\text {global }} \sim 1$; iii) $\mathrm{SFR}_{\mathrm{mm}} / \mathrm{SFR}_{\mathrm{UV}} \sim 50$. Below we discuss several scenarios that could help to reconcile these observations.

\subsection{Obscured stellar population}

The Sub-mm/radio emission could trace an obscured population of massive stars that could be undetectable at optical/NIR wavelengths. This has been suggested in the case of the host galaxy of the dark GRB 000210 (Gorosabel et al. 2003).

Given that $\mathrm{SFR}_{\mathrm{UV}} / \mathrm{SFR}_{\mathrm{mm}} \sim 0.02$, the probability that the progenitor belongs to the obscured population is $\sim 98 \%$ (assuming that the probability of making a GRB is only proportional to the SFR and not other parameters as e.g. the metallicity). However, opposite to the case of GRB 000210, GRB 000418 was not dark, so its progenitor either had to belong to the remaining $\sim 2 \%$ unobscured stellar population or the GRB destroyed the dust along the line of sight.

\subsection{Nuclear radio/sub-mm supernovae or AGN activity}

Another possibility is that the radio/sub-mm emission comes from a high nuclear radio supernovae rate $\left(>1 \mathrm{SN} \mathrm{yr}^{-1}\right)$ or from the activity associated with an AGN. Thus, the radio/mm emission would not invoke an optically hidden stellar population and the discrepancy between $\mathrm{SFR}_{\mathrm{UV}}$ and $\mathrm{SFR}_{\mathrm{mm}}$ would be naturally solved.

An appreciable amount of starbursts ( 40\%) contain compact radio cores (Kewley et al. 1999). These compact radio cores may be originated by obscured AGN or by complexes of luminous radio supernovae from an active nuclear starburst (Smith et al. 1998a). The GRB 000418 host galaxy would resemble the case of Arp 220, an active star forming galaxy (SFR 50-100 $M_{\odot} \mathrm{yr}^{-1}$ ) which shows a compact radio core (Smith et al. 1998b). The additional radio source detected by Berger et al. (2003) could also be related to AGN activity (e.g. the hot spot of a radio jet).

\section{Conclusions}

The analysis of optical/NIR observations presented in this paper confirms that the GRB 000418 host is a starburst galaxy. This result has been independently achieved by fitting synthetic and empirical templates to the photometric points. This conclusion is also consistent with the morphological information derived from the HST/STIS images, where the host is seen as a blue compact galaxy with no evidence for more widespread star formation. The more natural scenario would be a nuclear starburst that harbour a young population of stars where the GRB was originated. The reported offset of the afterglow respect to the galaxy nucleus $\left(0.023 \pm 0.064^{\prime \prime}\right.$; or a projected distance of 
$0.202 \pm 0.564 \mathrm{kpc}$ ) is consistent with this hypothesis (Bloom et al. 2002).

The synthetic SED fits are consistent with a young stellar population. The predicted host galaxy extinction, stellar age and star formation rate depend on the assumed extinction law. Two synthetic SED solutions are consistent with our photometric points: i) age $=59 \pm 6 \mathrm{Myr}, A_{V}^{\text {global }}=0.15 \pm 0.03$, $\mathrm{SFR}_{\mathrm{UV}} \sim 8 M_{\odot} \mathrm{yr}^{-1}$, progenitor mass $=\sim 7 M_{\odot}$; ii) age $=$ $4 \pm 1 \mathrm{Myr}, A_{V}^{\text {global }}=1.41 \pm 0.06, \mathrm{SFR}_{\mathrm{UV}} \sim 55 M_{\odot} \mathrm{yr}^{-1}$, progenitor mass $=\sim 60 M_{\odot}$. The second solution is not likely since it is inconsistent with the independent results obtained with the empirical templates, however it can not be completely discarded. In any case, both solutions are much easier to accommodate in the collapsar context (Woosley 1993; Paczyński 1998) than in the binary merging scenario (Eichler et al. 1989).

The global extinction of the host (defined as the averaged value of the ones obtained with the synthetic and empirical templates) is $\sim 0.4 \mathrm{mag}$, similar to the one measured along the line of sight to the afterglow. It is consistent with a rather homogeneous ISM, with no large density fluctuations, at least around the progenitor. This hypothesis would be supported by the smooth and symmetric radial profile inferred from the HST data. So the GRB might have not occurred in a extremely overdensed region of the host.

The inferred SFR $\mathrm{UV}_{\mathrm{UV}}\left(\sim 8 M_{\odot} \mathrm{yr}^{-1}\right)$ is two orders of magnitude lower than the one inferred from sub-mm/radio measurements.

Several ISM configurations have been proposed to explain this disagreement. In most of them an obscured population of massive stars (only visible in the sub- $\mathrm{mm} /$ radio range) is invoked. An alternative way to explain the radio emission could be the presence of a nuclear radio core originated by an AGN or by complexes of extremely luminous radio supernovae.

Acknowledgements. J. Gorosabel acknowledges J.-M. Miralles, M. Bolzonella and R. Pelló for helpful assistance with Hyperz. J. M. Castro Cerón acknowledges the receipt of a FPI doctoral fellowship from Spain's Ministerio de Ciencia y Tecnología. J. P. U. Fynbo acknowledges support from the Carlsberg Foundation. STH acknowledges support from NASA LTSA grant NAG5-9364. Part of the observations presented in this paper were obtained under the ESO Programmes 165.H-0464(I), 265.D-5742(C) (granted to the GRACE team) and 67.B-0611(A) (public data retrieved from ESO archive). Part of the data presented in this paper were acquired with ALFOSC, which is owned by the Instituto de Astrofísica de Andalucía (IAA) and operated at the Nordic Optical Telescope under agreement between IAA and NBIfAFG of the Astronomical Observatory of Copenhagen. We thank our anonymous referee by fruitful and constructive comments.

\section{References}

Andersen, M. I., Hjorth, J., Pedersen, H., et al. 2000, A\&A, 364, L54 Barnard, V. E., Blain, A. W., Tanvir, N. R., et al. 2003, MNRAS, 338, 1 Berger, E., Diercks, A., Frail, D. A., et al. 2001, ApJ, 556, 556 Berger, E., Cowie, L. L., Kulkarni, S. R., et al. 2003, ApJ, 588, 99 Bloom, J. S., Diercks, A., Galama, T. J., et al. 2000, GCN, 689 Bloom, J. S., Kulkarni, S. R., \& Djorgovski, S. G. 2002, AJ, 123, 1111
Bloom, J. S., Berger, E., Kulkarni, S. R., Djorgovski, S. G., \& Frail, D. A. 2003, AJ, 125, 999

Bolzonella, M., Miralles, J.-M., \& Pelló, R. 2000, A\&A, 363, 476

Bolzonella, M., Pelló, R., \& Miralles, J.-M. 2002, Hyperz manual (http://webast.ast.obs-mip.fr/hyperz/manual.html)

Bruzual, A. G., \& Charlot, S. 1993, ApJ, 405, 538

Calzetti, D., Armus, L., Bohlin, R. C., et al. 2000, ApJ, 533, 682

Chary, R., Becklin, E. E., \& Armus, L. 2002, ApJ, 566, 229

Christensen, L. 2002, Master Thesis, Copenhagen University, Astronomical Observatory

Christensen, L., Hjorth, J., Gorosabel, J., et al. 2003, A\&A, submitted

Dickey, J. M., \& Lockman, F. J. 1990, ARA\&A, 28, 215

Eichler, D., Livio, M., Piran, T., \& Schramm, D. N. 1989, Nature, 340, 126

Fitzpatrick, E. L. 1986, AJ, 92, 1068

Fruchter, A. S., Ferguson, H., Galama, T., et al. 1999, Cycle 8 HST proposal \# 8189

Fruchter, A. S., \& Hook, R. N. 2002, PASP, 114, 144

Gorosabel, J., Christensen, L., Hjorth, J., et al. 2001, to appear in From X-ray Binaries to Gamma-Ray Bursts, Proc. of Jan van Paradijs Memorial Symp., ed. E. P. J. van den Heuvel, L. Kaper, \& E. Rol (San Fransisco: ASP)

Gorosabel, J., Andersen, M. I., Hjorth, J., et al. 2002a, A\&A, 383, 112 Gorosabel, J., Fynbo, J. P. U., Hjorth, J., et al. 2002b, A\&A, 384, 11 Gorosabel, J., Christensen, L., Hjorth, J., et al. 2003, A\&A, 400, 127 Henden, A. 2000, GCN Circular, 662

Hjorth, J., Sollerman, J., Møller, P., et al. 2003, Nature, 423, 847 Holland, S., Fynbo, J. P. U., Hjorth, J., et al. 2001, A\&A, 371, 52

Kennicutt, R. C. 1992, ApJ, 388, 310

Kennicutt, R. C. 1998, ARA\&A, 36, 189

Kewley, L. J., Heisler, C. A., Dopita, M. A., \& Norris, R. P. 1999 , Ap\&SS, 266, 131

Kinney, A. L., Calzetti, D., Bohlin, R. C., et al. 1996, ApJ, 467, 38

Klose, S., Stecklum, B., Fischer, O., et al. 2000a, GCN, 645

Klose, S., Stecklum, B., Masetti, N., et al. 2000b, ApJ, 545, 271

Le Floc'h, E., Duc, P.-A., Mirabel, I. F., et al. A\&A, 2003, 400, 499

Lilly, S. J., Tresse, L., Hammer, F., Crampton, D., \& Le Fèvre, O. 1995, ApJ, 455, 108

Metzger, M., Fruchter, A., Masetti, N., et al. 2000, GCN, 733

Miller, G. E., \& Scalo, J. M. 1979, ApJS, 41, 513

Oke, J. B. 1990, AJ, 99, 1621

Paczyński, B. 1998, ApJ, 494, L45

Persson, S. E., Murphy, D. C., Krzeminski, W., Roth, M., \& Rieke, M. J. 1998, AJ, 116, 2475

Portinari, L., Chiosi, C., \& Bressan, A. 1998, A\&A, 334, 505

Prévot, M. L., Lequeux, J., Prévot, L., Maurice, E., \& Rocca-Volmerange, B. 1984, A\&A, 132, 389

Salpeter, E. E. 1955, ApJ, 121, 161

Scalo, J. M. 1986, Fundam. Cosmic Phys., 11, 1

Schechter, P. 1976, ApJ, 203, 297

Schlegel, D. J., Finkbeiner, D. P., \& Davis, M. 1998, ApJ, 500, 525

Seaton, M. J. 1979, MNRAS, 187, 73

Smith, H. E., Lonsdale, C. J., \& Lonsdale, C. J. 1998a, ApJ, 492, 137

Smith, H. E., Lonsdale, C. J., Lonsdale, C. J., \& Diamond, P. J. 1998b, ApJ, 493, L17

Sokolov, V. V., Fatkhullin, T. A., Castro-Tirado, A. J., et al. 2001, A\&A, 372, 438

STScI Instrument Handbook, 2000 STScI

Vreeswijk, P. M. 2002, Ph.D. Thesis, Univ. of Amsterdam

Woosley, S. E. 1993, ApJ, 405, 273

Xilouris, E. M., \& Papadakis, I. E. 2002, A\&A, 387, 441 